\title{
Perceptions of legislation relating to the sharing of genomic biobank results with donors-a survey of BBMRI-ERIC biobanks
}

\author{
Minna Brunfeldt ${ }^{1} \cdot$ Harriet Teare $^{2} \cdot$ Sirpa Soini $^{3} \cdot$ Helena Kääriäinen $^{1}$
}

Received: 19 April 2017 / Revised: 8 September 2017 / Accepted: 7 November 2017 / Published online: 12 January 2018

(c) European Society of Human Genetics 2018

\begin{abstract}
Biobanks accumulate huge amounts of research findings, including participants' genomic data. Increasingly this leads to biobanks receiving research results that could be of clinical significance to biobank participants. The EU Horizon 2020 Project 'Genetics Clinic of the Future' surveyed European biobanks' perceptions of the legal and regulatory requirements for communicating individual research results to donors. The goal was to gain background knowledge for possible future guidelines, especially relating to the consent process. The Survey was implemented using a web-based Webropol tool. The questionnaire was sent at the end of 2015 to 351 European biobanks in 13 countries that are members of BBMRI-ERIC (Biobanking and Biomolecular Resources Research Infrastructure-European Research Infrastructure Consortium). Seventy-two biobanks responded to the survey, representing each of the 13 BBMRI Member States. Respondents were mainly individuals responsible for the governance of biobanks. The replies indicate that the majority of the respondents thought that their national legislation allowed them to contact participants to communicate results, and that research participants had the right to request their results. However, respondents' understanding of their national legislation varied even within member states. Our results indicate that legislation applied to biobanks in many countries may be scattered and difficult to interpret. In BBMRI-ERIC, there is an ongoing discussion about the need for European recommendations on sharing genomic biobank results with donors, which may pave the way for more coherent global guidelines. Our results form a basis for this work.
\end{abstract}

\section{Introduction}

Patients with a suspected genetic condition or predisposition may undergo a genome-wide examination as an initial genetic test. This test may uncover results relevant to a patient's health that are unrelated to the initial inquiry. There has been a significant discussion about whether patients should be informed of all results that might be relevant for their health or whether they should only receive those directly related to their symptoms. In 2013, the American College of Medical Genetics and Genomics (ACMG) issued recommendations for reporting incidental findings in 56

Minna Brunfeldt

minna.brunfeldt@thl.fi

1 Genomics and Biomarkers Unit, National Institute for Health and Welfare, Haartmaninkatu 8, Helsinki, Finland

2 Nuffield Department of Population Health, University of Oxford, Oxford, United Kingdom

3 Helsinki Biobank, HUS, Helsinki, Finland "actionable" genes as part of clinical exome sequencing (ES) or whole-genome sequencing (WGS). These recommendations do not relate to genomic sequencing performed in a research setting [1, 2]. The European Society of Human Genetics has continued this discussion stating that developing a possible European consensus still needs further consideration [3]. More recently, the UK 100,000 genomes project, which is at the borderline between research and the clinic, has suggested reporting a reduced list of 10 wellknown actionable genes. In addition, some argue that variant data predicting future multifactorial diseases would also be important for patients [4].

Biobanks are infrastructures created for collecting and storing human biological material and data for the purposes of future research. Genomic data including ES and WGS data are already accumulating in biobanks even though WGS is not yet widely used in the clinical settings. As biobanks collect and analyze samples for research purposes and are not directly connected to healthcare services, the results from these collections cannot automatically be included in the hospital files or revealed to the clinicians 
treating the individuals if and when they become patients [5]. It has been asked whether the donors themselves should be notified about results that could guide their healthcare. However, at present there are no shared protocols and practices that outline how to feed genomic results from a research into a healthcare setting. The OECD Guidelines on Human Biobanks and Genetic Research Database recognizes the importance of analyzing genomic information together with other health and personal data to understand multifactorial diseases [6]. The Guidelines specifically state that biobanks should have a clearly articulated policy on whether, and how, to return the accumulating individual genomic results to donors.

The Genetics Clinic of the Future (GCOF) is an EUfunded project focusing on developing ideas and tools for effective, patient-driven, and sustainable use of genomic data in future healthcare. The GCOF brings together expertise in clinical genetics, ethics, biolaw, health policy, patient and citizen representation, and data sharing (link to GCOF page www.geneticsclinicofthefuture.eu). One of the aims of the GCOF was to explore the policies, practices, and experiences in sharing research data with donors. This was achieved by surveying (using a web-based survey) European biobanks participating in the BBMRI-ERIC (Biobanking and Biomolecular Resources Research Infrastructure-European Research Infrastructure Consortium), because they have already gathered a huge amount of genomic data and the issue of sharing data therefore arises as part of their everyday work.

The Survey was conducted at the end of 2015. In this paper, we focus on describing the diversity of the European legislation relating to sharing research results with donors as experienced by the respondents, and how this is reflected in the consenting practices of biobanks. The expectation was that this data would initiate discussion on the possibility of having common and harmonized practices in Europe for sharing results with donors, which could be introduced in the consent process.

\section{Materials and methods}

Informal in-depth discussions among the interdisciplinary GCOF consortium (at two project meetings and subsequently via email) formed the basis for preparation of the questionnaire. All the comments were collected and taken into account.

The draft electronic questionnaire was piloted by sending the link to a group of participants at the HandsOn: Biobanks 2015 Conference (HOBB) on 29-31 July 2015 in Milan, Italy. These participants identified themselves as biobank directors, heads, managers or similar, and were therefore deemed to provide good representation of the intended survey recipients. Their opinions and suggestions were collected soon after the conference. In response to their feedback, the questionnaire was reformed, and the new draft was sent for final comments to the members of the GCOF consortium.

The questionnaire was formulated using the Webropol online survey and analysis tool (www.webropol.fi). On 28 October 2015, the questionnaire was sent to all biobanks listed at that time on the BBMRI-ERIC Directory 1.0 (http://bbmri-eric.eu/bbmri-eric-directory). Since the survey was implemented, the number of BBMRI Member States has increased to 19 (as of 31 August 2016). The questionnaire was conducted in English for all the respondents. Reminders were sent on 9th of November, 17th of November, and 2nd of December 2015. The BBMRI-ERIC network advertised the survey through their web page and Newsletter.

\section{Results}

In the BBMRI-ERIC Directory 1.0, there were 511 contact addresses for biobanks. After the removal of duplicated addresses, the questionnaire was sent to the remaining 380 biobanks in 13 countries, of which 24 addresses did not work and five biobanks replied that they did not have genomic data, human samples, or that they were only small cohorts. This left 351 biobanks in total. Seventy-two replies were received (response rate 21\%), which represented all 13 BBMRI-ERIC countries at that time (Table 1). The responding biobanks represented a variety of different human collections (Fig. 1). Nearly all respondents identified themselves as biobank directors, heads, managers or similar.

Table 1 The number of the respondents by country

\begin{tabular}{lll}
\hline Member state & Respondents & Reply $(\%)$ \\
\hline Czech Republic & $4 / 5$ & 80 \\
Italy & $18 / 64$ & 28 \\
France & $10 / 70$ & 14 \\
Malta & $1 / 1$ & 100 \\
Belgium & $10 / 14$ & 71 \\
Austria & $1 / 3$ & 33 \\
Netherlands & $8 / 107$ & 7 \\
Finland & $3 / 8$ & 38 \\
Poland & $1 / 2$ & 50 \\
Germany & $5 / 10$ & 50 \\
Estonia & $1 / 1$ & 100 \\
Greece & $1 / 1$ & 100 \\
Sweden & $8 / 65$ & 12 \\
Together & $72 / 351$ & 21 \\
\hline
\end{tabular}




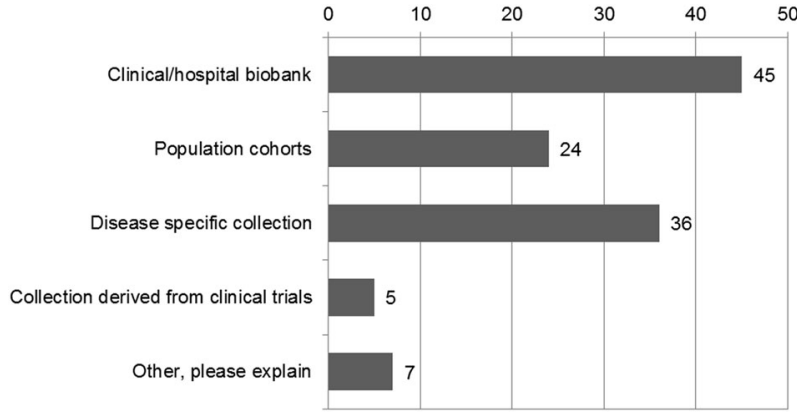

Fig. 1 Replies to the question "What type of a biobank do you represent?" $(n=72)$. The respondents were allowed to choose several options

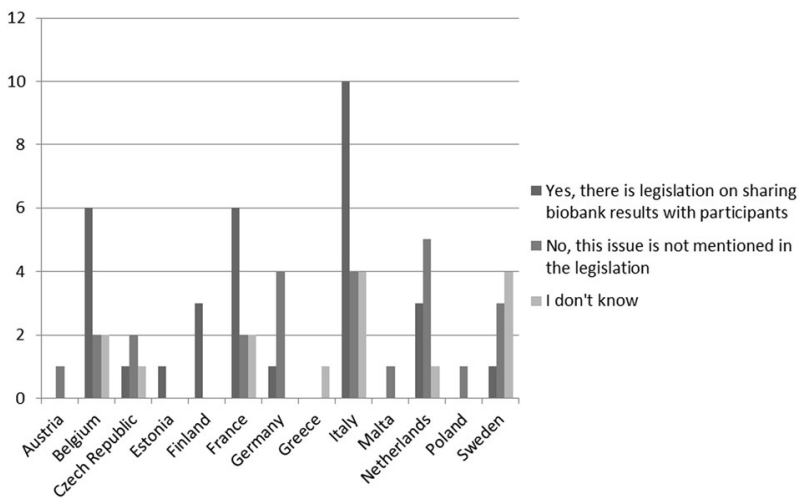

Fig. 2 Replies to the question "Do your national laws and/or other regulations explicitly mention the possibility of sharing individualspecific results with participants in the biobank?", between countries and internally $(n=72)$

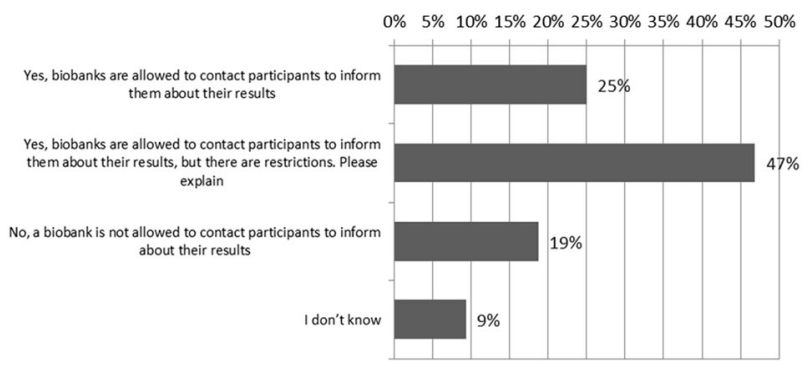

Fig. 3 Replies to the question "Do your national laws and/or other regulations allow biobanks to contact participants to inform them about results concerning their health?" $(n=32)$

Four specific questions were used to explore respondents' understanding of the legislation or other national regulation relating to sharing individual (genomic) results with donors (Figs. 2, 3, 4, and 5). When asked about whether national legislation or other regulation explicitly mentioned the return of results to donors, $44 \%$ replied that it was mentioned in the legislation, 35\% said that it was not mentioned, and $21 \%$ did not know. The replies varied between respondents from the same country as shown in Fig. 2.

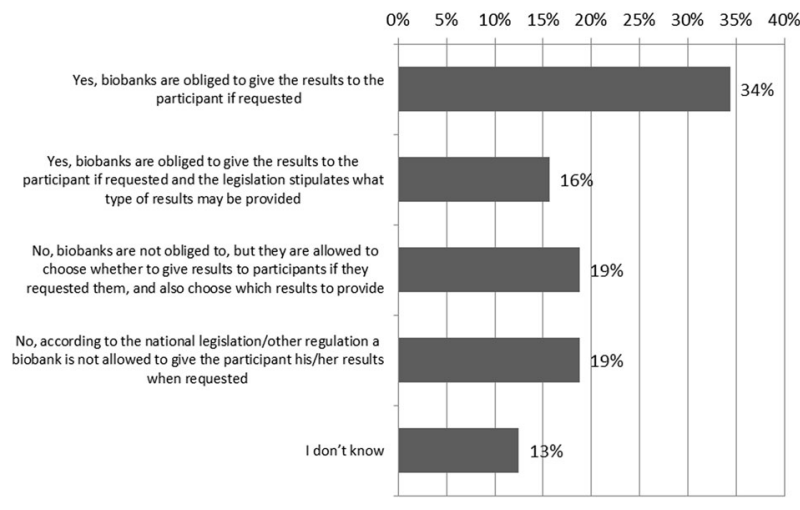

Fig. 4 Replies to the question "Do your national laws and/or other regulations oblige biobanks to give participants their results if they request them?" $(n=32)$

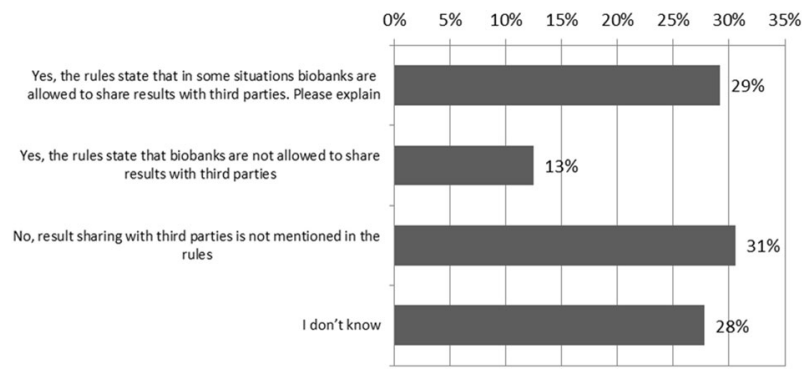

Fig. 5 Replies to question "Do your national laws and/or other regulations stipulate whether biobank results may or may not be shared with third parties?" $(n=72)$

Respondents were asked whether biobanks are allowed to actively contact participants to share genomic results, if they are obliged or allowed to give results if the participant requests them, and, finally, if they are allowed (in any circumstances) to give the results to third parties. The responses are presented in Figs. 3, 4, and 5.

Of the 15 biobanks (47\%) that replied that the legislation/regulation allowed the biobank to contact donors to report (some) results of genetic/genomic tests, but with restrictions (Fig. 3), each contributed a free text answer to explain. Six out of 15 stated that this had to have been agreed and/or specified in the consent process, $1 / 15$ mentioned restrictions relating to the possible implications of the results to the health of the individual, and $2 / 15$ said that there had to be an approval by an ethics committee.

Of the 21 biobanks (29\%) that replied that in some situations biobanks are allowed to share the results with third parties, $2 / 21$ stated that it is possible to share the results with donor's relatives or family, if it is essential for their health; 7/21 mentioned that the participants are able to specify this in the consent form.

We also wanted to know how biobanks discuss the possibility of sharing results with the participants in the 


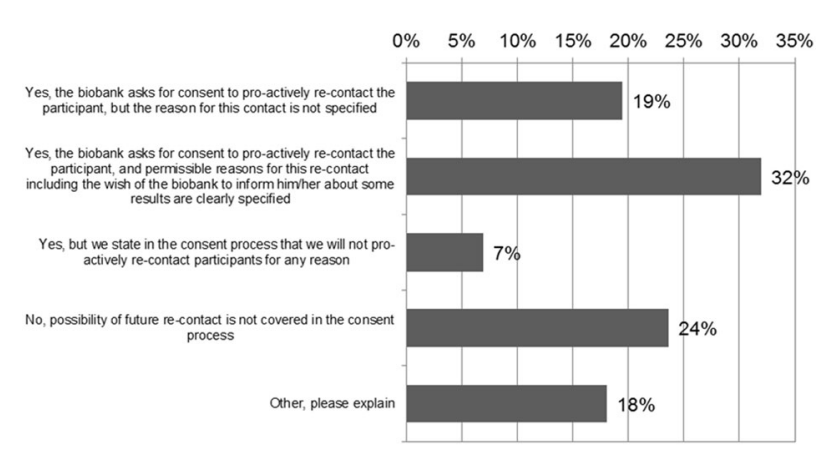

Fig. 6 Replies to question "In the consent process of your biobank, do you discuss the option of re-contacting the participant?" $(n=72)$

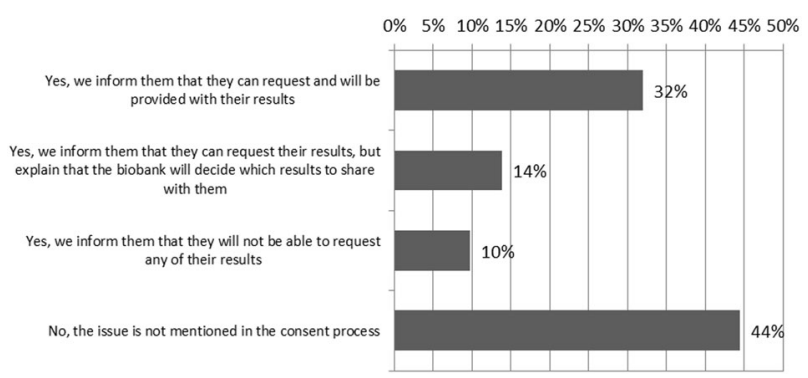

Fig. 7 Replies to question "In the consent process of your biobank, do you discuss the possibility of the participant requesting results from the biobank?" $(n=72)$

course of the consent process. This was explored across two questions (Figs. 6, 7).

\section{Discussion}

BBMRI-ERIC aims to increase the efficacy and excellence of European bio-medical research by facilitating access to quality-defined human health/disease-relevant biological resources (http://www.bbmri-eric.eu/). The 72 biobanks participating in the survey represented each of the EU countries that were members of BBMRI-ERIC at the time that the survey was distributed. As members of BBMRIERIC, all the biobanks were part of the research infrastructure. Most of the biobanks replied that they are based on clinical collections (21 biobanks, 29\%) or clinical and disease-specific collections (19 biobanks, 26\%). The third common combination was a clinical/population/diseasespecific collection.

Given that respondents to the questionnaire were largely involved in the governance of the biobanks, it might have been anticipated that they would have been aware of the current legislation in their country. It was somewhat surprising, therefore, that $21 \%$ of the respondents were not aware if there were national laws or regulations mentioning the possibility of sharing individual results with biobank participants. In addition, respondents representing the same country sometimes had a different understanding of the national regulation. There may be several explanations for this confusion. The national legislation may be scattered in different laws relating to, for instance, patient rights, data protection, and medical research in a way that is difficult to comprehend. Also internationally, the regulatory scene is very complex in the biomedical field with transnational laws, ethical codes, guidelines, and other policy papers. Non-lawyers may find it hard to understand what the legal obligations are [7].

According to our respondents, $72 \%$ think that the national legislation allows the biobanks to contact their participants in order to inform them about their results. On the other hand, 50\% answered that research participants have the right to request their results. Yet, many others claimed that these approaches are forbidden. As one of the aims of BBMRI-ERIC is to harmonize biobank procedures as much as possible in the EU, these apparent discrepancies in the legislation need careful consideration when forwarding this intention.

Over 130 leading healthcare, research, and disease advocacy organizations in 40 countries have signed a letter of intent to work toward the formulation of a Global Alliance for Genomics and Health (GA4GH) [8]. The goal of the collaboration is (1) to enable open standards for interoperability of technology platforms for managing and sharing genomic and clinical data, (2) to provide guidelines and harmonized procedures for privacy and ethics internationally, and (3) to engage stakeholders to encourage responsible sharing of data and methods [9]. These developments would speed up the progress of genomic research and provide more options for returning results to participants.

In biobanking, as with all medical research involving human participants, one of the key governance tools to support patients and participants is the informed consent process [10]. In research involving biobank samples, results from genome sequencing may have important consequences for participants. In spite of this, a remarkable number of biobanks replied that they do not discuss return of results in their consent process (Figs. 6 and 7).

Three out of four of the authors of this paper are from Finland. In Finland, there is a relatively new Biobank Act (688/2012) which stipulates most of the issues relating to the governance of biobanks. As far as we know, there is no comparable comprehensive biobank law in any of the other BBMRI-ERIC countries with the exception of Estonia. The three Finnish biobanks that replied to our survey had identical answers that demonstrated clear awareness of the legislation. We believe that this is partly because Finland has a specific biobank law instead of having the regulation scattered through different laws. 
One issue mentioned by several respondents in the openended parts of the questionnaire was a concern about the quality of the results. As biobank results are gathered from different research laboratories, the quality is not always reliable compared with accredited clinical laboratories. According to our survey, this problem has not been solved in most of the biobanks. In biobank research, samples from different biobanks are often combined and analyzed together in one center. If such results are considered "reliable" in one biobank but as "needing confirmation" in another, then this will give the donors a confusing message.

It has been increasingly demonstrated that biobanks should inform genomic results to donors [11] but there are only a few concrete examples, partly due to many legal, financial, organizational, and societal challenges that need to be tackled first [12]. Some biobanks have tested a model for contacting donors to inform them about certain results, as described by Haukkala et al. [13] and Leitsalu et al. [14]. These models have included a first contact with very general information provided about possibly actionable results, new consent stating willingness to hear genetic results, requests for a new sample with re-testing in a clinical laboratory, and finally informing (and guidance for healthcare) only after the new test result. Both examples are from a research setting where ways of reporting results were being investigated. These studies concerned a relatively small number of people. To introduce such an approach on a large scale would be a tremendous financial and logistical challenge. Most biobanks do not have resources for routinely approaching donors and offering re-testing in case of possibly actionable results. Common criteria on actionable results are hard to draft and cannot be stipulated by law. Lack of qualified staff is also a problem, since clinical geneticists and/or genetic counselors are few and primarily needed in clinical practice. Thus, practical challenges outweigh the legal ones [12]. When biobanks start to concretely plan the processes of returning results, different attempts to solve the practical obstacles will need to be carefully investigated.

Our research had some limitations. One of them is that only $21 \%$ of the biobanks replied and we have no data on the non-respondents or their reasons for not responding. Thus, we think that this is a descriptive study that presents the impression that we got from the Survey but cannot be considered strictly quantitative. Also only one respondent replied on behalf of one biobank, and even though the respondents were mainly stewards of biobanks, there is still a possibility that another person would have provided supplementary information. In addition, the definition of a biobank is not unambiguous and we cannot be sure that the responding biobanks are all the presumed similar research infrastructures.

\section{Conclusion}

BBMRI-ERIC biobanks constantly collaborate in various research projects. Ideally, practices of returning results and discussing the issue in the consent processes should be as harmonized as possible in Europe to allow for similar treatment of donors when large cohorts are combined, or for international research endeavors. However, as long as the national regulation is so diverse, this may be impossible to achieve and challenges in international collaboration will still remain. BBMRI-ERIC provides an excellent forum to continue discussions aimed at harmonizing practices, where possible, relating to all aspects of biobank research, including sharing genomic results with individual donors. We believe that our results will stimulate these discussions.

\section{Compliance with ethical standards}

Conflict of Interest The authors declare that they have no conflict of interests.

\section{References}

1. Green R, Berg J, Grody W, et al. ACMG recommendations for reporting of incidental findings in clinical exome and genome sequencing. Genet Med. 2013;15:565-74.

2. Kalia SS, Adelman K, Bale SJ, et al. Recommendations for reporting of secondary findings in clinical exome and genome sequencing, 2016 update (ACMG SFv2.0): a policy statement of the American College of Medical Genetics and Genomics. Genet Med. 2017;19:249-55.

3. Hehir-Kwa JY, Claustres M, Hastings RJ, et al. Towards a European consensus for reporting incidental findings during clinical NGS testing. Eur J Hum Genet. 2015;23:1601-6.

4. Caulfield M, Davies J, Dennys M, et al. The 100,000 Genomes Project Protocol, Genomics England. 109pp. https://www. genomicsengland.co.uk/wp-content/uploads/2015/03/GenomicEngla ndProtocol_030315_v8.pdf. 2017.

5. Prince AE, Conley JM, Davis AM, et al. Automatic placement of genomic research results in medical records: do researchers have a duty? Should participants have a choice? J Law Med Ethics. 2015;43:827-42.

6. OECD Guidelines for Human Biobanks and Genetic Research Databases. 2009. 47pp.

7. Soini S. Biobanks as a central part of the Finnish growth and genomic strategies: how to balance privacy in an innovation ecosystem. pg 33. J Law Med Ethics. 2016;44:24-34.

8. Knoppers BM. International ethics harmonization and the global alliance for genomics and health. Genome Med. 2014;6:13.

9. Knoppers BM, Zawati H, Sénécal K. Return of genetic testing results in the era of whole-genome sequencing. Nat Rev Genet. 2015;16:553-9.

10. Caulfield T, McGuire AL, Cho M, et al. Research ethics recommendations for whole-genome research: consensus statement. PLoS Biol. 2008;6:e73.

11. Wolf S, Crock BN, Van Ness B, et al. Managing incidental findings and research results in genomic research involving biobanks and archived data sets. Genet Med. 2012;14:361-84. 
12. Budin-Ljøsne I, Mascalzoni D, Soini S, et al. Feedback of individual genetic results to research participants: is it feasible in Europe. Biopreserv Biobank. 2016;14:241-8.

13. Haukkala A, Kujala E, Alha P, et al. The return of unexpected research results in a biobank study and referral to health care for heritable long QT syndrome. Public Health Genom. 2013;16:241-50.

14. Leitsalu L, Alavere H, Jacquemont S, et al. Reporting incidental findings of genomic disorder-associated copy number variants to unselected biobank participants. Future Med. 2016;13:303-14. 